\title{
INTERLACING PROPERTY OF ZEROS OF EIGENVECTORS OF SCHRÖDINGER OPERATORS ON TREES
}

\author{
FRANÇOIS CHAPON
}

\begin{abstract}
We prove an analogue for trees of Courant's theorem on the interlacing property of zeros of eigenfunctions of a Schrödinger operator. Let $\Gamma$ be a finite tree, and $\mathcal{A}$ a Schrödinger operator on $\Gamma$. If the eigenvectors of $\mathcal{A}$ are ordered according to increasing eigenvalues, and the vertices corresponding to zero coordinates are of degree at most two, then the zeros of the linear extensions of eigenvectors have the interlacing property.
\end{abstract}

\section{INTRODUCTION}

The famous Courant's theorem [6] about nodal domains of eigenfunctions of differential operators states that:

Theorem (Courant's theorem). Let $L$ be a self-adjoint second order elliptic operator on a domain $G$ with arbitrary boundary conditions. If its eigenfunctions are ordered according to increasing eigenvalues, then the nodes of the $n$-th eigenfunction $u_{n}$ divide the domain into no more than $n$ subdomains.

The subdomains defined in the theorem are called nodal domains and are the connected components of the complement of the nodal set $\left\{x \mid u_{n}(x)=0\right\}$, which are separated by the nodes, or zeros, of eigenfunctions of $L$.

If $L$ is a Schrödinger operator on $G=[a, b] \subset \mathbb{R}$, that is $L=\mathcal{L}+V$ where $\mathcal{L}$ is the Laplacian and $V$ some potential, Courant's theorem becomes: The nodes of the $n$-th eigenfunction $u_{n}$ divide $G$ into exactly $n$ nodal domains. Besides, the zeros of eigenfunctions of $L$ have an interlacing property: Between two zeros of $u_{n}$, there is exactly one zero of $u_{n+1}$.

Analogues of Courant's result on graph have recently received increasing attention, both in the mathematical and in the physical literature, and one may cite for instance the book [4 for a good introduction to this subject, and [7] for some historical comments. In [7, the authors Davies et al. prove a upper bound discrete nodal theorem on graphs. Contrary to the situation when $G$ is a manifold, since a function on a graph is only defined on the set of vertices, an eigenfunction can change its sign without passing through zero, hence the nodes are not well defined. So Davies et al. introduce the notion of strong sign graph, which is a connected set of vertices on which the eigenvector has the same sign, and prove the analogue of Courant's result on the maximal number of sign graphs of eigenvectors of a Schrödinger operator. Their proof is based on Courant's minimax theorem, see [6], and some straightforward algebra. In [3], Bıyıkoğlu proves an analogue of

2010 Mathematics Subject Classification. 05C05; 05C50; 15A18.

Key words and phrases. Schrödinger operator, tree, discrete nodal theorem, interlacing property. 
Courant's theorem on trees under a certain genericity condition: he proves that if $u$ is an eigenvector associated to the $n$-th ordered eigenvalue $\lambda_{n}$ and without a vanishing coordinate, then $u$ has exactly $n$ strong sign graphs and also gives a computational algorithm to find an eigenvector with minimum number of sign graphs when allowing multiplicity. In [2], Berkolaiko proves also a nodal theorem on graphs under the same condition (both in the discrete and metric case). More precisely he gives a lower and upper bound of the nodal domains count of eigenfunctions of a generalized Laplacian on graphs and in particular recovers Bıyıkoğlu's result on trees. Recently, Xu and Yau [13] give a uniform proof of the lower and upper bounds of strong nodal domains on possibly disconnected graph, hence extending the results of [2].

On the physical level, the study of nodal domains appears in many different areas, such as quantum chaos, isospectral properties, or percolation theory. One may cite for instance [1] where several algorithmic and analytic methods for counting nodal domains are presented, [10] for the connection between nodal domains count on manifolds and geometrical content of the domain, [12] for an example of isospectral graphs with the same nodal count sequence, or [5] for nodal domains statistics as a criterion for quantum chaos.

Here, we are interested in the analogue of Courant's interlacing property for Schrödinger operators on finite trees. Since on a tree there is a unique path connecting two vertices, we can extend a function by linearity on the edges, which allows us to define the zeros of an eigenfunction not only on vertices. This idea of geometric realization of a tree goes back to Friedman [9]. To assure that the zeros of the eigenfunctions are well-defined, we make the assumption that the zeros coordinates of eigenvectors are of degree at most two, which implies that the eigenvalues are simple and that the corresponding eigenvectors are without a zero graph. The situation becomes then very similar to what happens in the real unidimensional case, and we will prove in Theorem 4.1 the interlacing property of the zeros of the linear extension of eigenvectors of a Schrödinger operator. In particular we recover the exact nodal count already known for trees [3, 2]. The proof of these facts follows the lines of the classical proof of Courant [6] and are based on an analogue of Green's formula and on the discrete nodal upper bound proved in [7. Note that, inspired by our method, Griffing, Lynch and Stone prove in [1] the interlacing property of the zeros of harmonic functions of the Laplacian, defined as the extension of the eigenvectors of the Laplacian of a subgraph given by the Schur complement of the Laplacian with respect to the pendant vertices.

The following is organized as follows. In section 2, we introduce the notations, present the tree geometric realization allowing to consider linear extension of functions on edges, and discuss some examples. Section 3 is devoted to prove the Green's formula on trees, and finally in section 4 we prove the Courant's nodal theorem on the interlacing property of zeros of eigenvectors of a Schrödinger operator on a tree.

\section{Definitions And nOtations}

2.1. Schrödinger operators. Let $\Gamma=(V, E)$ be a finite tree, where $V$ is the set of vertices and $E$ the set of edges, with $|V|=N$, and $|E|=N-1$. Recall that a tree is a connected graph without cycles. This means that on a tree, any two points (possibly on the edges) are connected by a unique path. We note for $x, y \in V, x \sim y$ if there is an edge connecting $x$ and $y$, and $x$ and $y$ are said to be neighbors, or 
adjacent. The degree of a vertex $x$ is the number of edges connecting $x$. We choose some vertex with only one neighbor and call it the root of the tree. This gives an orientation of the edges, the positive orientation being the direction connecting the root and the vertices. We note $(x, y)$ the edge starting from $x$ and ending at $y$. We consider weighted trees, that is there is a function $c: V \times V \rightarrow \mathbb{R}$ such that

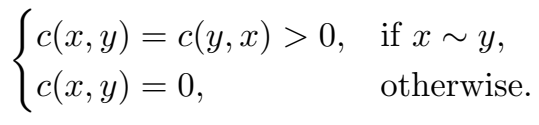

For $x \sim y$, let $l(x, y)=\frac{1}{c(x, y)}$. As in [9], $l(x, y)$ is called the length of the edge connecting $x$ and $y$. Define $L^{2}(V)$ the space of functions on $V$ endowed with the scalar product

$$
\langle u, v\rangle_{V}=\sum_{x \in V} u(x) v(x),
$$

for $u, v$ functions on $V$. Define also $L^{2}(E)$ the space of functions $f$ on $E$ such that $f(x, y)=-f(y, x)$ for all edges $(x, y) \in E$, endowed with the scalar product

$$
\langle f, g\rangle_{E}=\sum_{e \in E} f(e) g(e)=\frac{1}{2} \sum_{x \in V} \sum_{y \in V, y \sim x} f(x, y) g(x, y),
$$

for $f, g \in L^{2}(E)$, where we use the notation $f(x, y)=f((x, y))$ for functions on $E$ for clarity. The derivative operator $\partial: L^{2}(V) \rightarrow L^{2}(E)$ is defined by

$$
\partial u(x, y)=c(x, y)^{1 / 2}(u(x)-u(y)),
$$

for $u \in L^{2}(V)$, and its adjoint $\partial^{*}: L^{2}(E) \rightarrow L^{2}(V)$ is then given by

$$
\partial^{*} g(x)=\sum_{y \sim x} c(x, y)^{1 / 2} g(x, y)
$$

for $g \in L^{2}(E)$.

Let $\mathcal{L}=\partial^{*} \partial: L^{2}(V) \rightarrow L^{2}(V)$. Then $\mathcal{L}$ is called the Laplacian on $\Gamma$, and for $f \in L^{2}(V)$, we have for all $x \in V$,

$$
\mathcal{L} f(x)=\sum_{y \in V, y \sim x} c(x, y)(f(x)-f(y)) .
$$

Note that $\mathcal{L}$ is a self-adjoint operator on $L^{2}(V)$. If we see $f$ as a vector in $\mathbb{R}^{N}$, then $\mathcal{L}$ can be seen as a $N \times N$ symmetric matrix whose nonzero entries are given by

$$
\begin{cases}\mathcal{L}_{x y}=-c(x, y), & \text { for } x \sim y, \\ \mathcal{L}_{x x}=\sum_{y \sim x} c(x, y), & \text { on the diagonal. }\end{cases}
$$

We recall now the notion of Schrödinger operators. Let $r: V \rightarrow \mathbb{R}$ be some function on $V$, which plays the role of some potential. Define $\mathcal{A}=\mathcal{L}+r$. Then, for all $f \in L^{2}(V)$, and all $x \in V$,

$$
\mathcal{A} f(x)=\sum_{y \in V, y \sim x} c(x, y)(f(x)-f(y))+r(x) f(x) .
$$

The operator $\mathcal{A}$ is called a Schrödinger operator, or a generalized Laplacian, on $\Gamma$. As for the Laplacian, $\mathcal{A}$ can be seen as a $N \times N$ symmetric matrix, with non-positive off-diagonal elements. 
In the sequel, we will note $\lambda_{i}, i=1, \ldots, N$, the (real) eigenvalues of $\mathcal{A}$ ordered such that

$$
\lambda_{1} \leq \lambda_{2} \leq \cdots \leq \lambda_{N}
$$

and such that the eigenspaces are orthogonal with respect to $\langle\cdot, \cdot\rangle_{V}$. It is well known that, by the Perron-Frobenius theorem, $\lambda_{1}$ is simple and the first eigenvector can be chosen everywhere positive, see [7. By orthogonality, any eigenvector associated with an eigenvalue different from $\lambda_{1}$ must then changes sign on $V$.

2.2. Discrete nodal theorem. We introduce now the notion of sign graphs of a function on $V$ as defined in [7.

Definition 2.1. Let $u$ be a function on $V$. A strong positive (resp. negative) sign graph of $u$ is a maximal subtree $S$ of $\Gamma$ with $u(x)>0$ (resp. $u(x)<0)$, for all vertices $x$ of $S$.

Then the theorem of Davies et al. [7, which is an analogue on graph of the Courant's nodal theorem, states that:

Theorem $2.2([7])$. Let $\lambda_{1}, \ldots, \lambda_{N}$ be the ordered eigenvalues of a Schrödinger operator on $\Gamma$. Suppose $\lambda_{n}$ is of multiplicity $r$. Then any eigenvector corresponding to $\lambda_{n}$ has at most $n+r-1$ strong sign graphs.

Let $u$ be an eigenvector of $\mathcal{A}$ with eigenvalue $\lambda$. Let $x$ be a zero vertex of $u$, i.e. $u(x)=0$. Then, $\mathcal{A} u(x)=0$, and we have

$$
\sum_{y \sim x} c(x, y) u(y)=0 .
$$

Since $c(x, y)>0$ for all $x \sim y$, two cases are possible: we have either $u(y)=0$ for all $y \sim x$, and $x$ is said to belong to a zero graph, or $x$ is adjacent to both strict signs. For our context of Schrödinger operators, we make the following assumption.

Assumption (A). For all $i=1, \ldots, N$, denote by $u_{i}$ an eigenvector associated with $\lambda_{i}$. Let $s_{k}^{(i)}$ be the number of vertices corresponding to the zeros of $u_{i}$ and of degree $k$. Then $s_{k}^{(i)}=0$ for all $k \geq 3$ and all $i=1, \ldots, N$, that is any zero vertex is of degree at most two.

This assumption implies that if there is a zero vertex, it is of degree exactly two. Indeed, if there is a zero graph, then since a tree is connected it has to end with a zero vertex adjacent to both strict signs, thus of degree at least three, hence contradicting the assumption. A zero vertex has then exactly two adjacent vertices of both strict signs and no end vertex can be a zero vertex. By a result of Fiedler, see Theorem 2.4 and Corollary 2.7 in [8], this is equivalent of saying that all eigenvalues are simple and the corresponding eigenvectors are without a zero graph. This will insure that the zeros of the linear extension of the eigenvectors are well defined, see next subsection.

Under assumption (A), the theorem of Davies et al. becomes then: any eigenvector associated with $\lambda_{n}$ has at most $n$ strong sign graphs. Note that Biylkoğlu [3] (see also [2]) proves under the assumption that all eigenvectors are without vanishing coordinates, that any eigenvector associated with $\lambda_{n}$ has exactly $n$ strong sign graphs. 
2.3. Geometric realization. As introduced by Friedman [9] (see also [4]), we consider a geometric realization of $\Gamma$. An edge $(x, y)$ can be identified with the real interval $[0, l(x, y)]$. A function $u$ defined on $V$ can then be extended on the edges by linearity. Let us call $\tilde{u}_{x y}$ this extension on the edge $(x, y)$. We have then

$$
\tilde{u}_{x y}(t)=\frac{u(y)-u(x)}{l(x, y)} t+u(x), \quad \text { for } t \in[0, l(x, y)] .
$$

We then denote by $\tilde{u}$ the linear extension of $u$ on each edge.

We introduce now the correspondence between strong sign graphs of eigenvectors of $\mathcal{A}$ and the nodal domains of their respective linear extensions.

Definition 2.3. Let $u$ be a function on $V$, and $\tilde{u}$ its linear extension on edges. A nodal domain of $\tilde{u}$ is a maximal connected path on which $\tilde{u}$ does not vanish.

Since a tree is connected, this notion is well defined. For $u$ an eigenvector of $\mathcal{A}$, the different nodal domains of $\tilde{u}$ are then separated by the zeros of $\tilde{u}$ which are well defined by assumption (A) and possibly on the edges. Note that a nodal domain of $\tilde{u}$ can also be seen as a subtree of $\Gamma$ with some incomplete edges.

Let $G$ be a strong sign graph of $u$. Let $(x, y)$ be an edge with $x \in G$ and $y \in V \backslash G$. Then $u(x) u(y) \leq 0$ on $(x, y)$, otherwise $y$ would be in $G$. This implies that $\tilde{u}$ must vanish on the interval $] 0, l(x, y)]$. Hence, $G$ defines uniquely a nodal domain $\tilde{G}$ of $\tilde{u}$, because, since $\tilde{u}$ is linear on any edge, we cannot have two zeros on the same edge, and a nodal domain contains at least one vertex. So, there is a one-to-one correspondence between strong sign graphs of $u$ and nodal domains of its linear extension $\tilde{u}$.

2.4. Some examples. First let us consider the simple example of a linear graph with weights 1,1 on both edges. The Laplacian is given by

$$
\mathcal{L}=\left(\begin{array}{ccc}
1 & -1 & 0 \\
-1 & 2 & -1 \\
0 & -1 & 1
\end{array}\right)
$$

The eigenvalues are 0,1 and 3 and are simple (recall that it is a well known fact that the eigenvalues of tridiagonal symmetric matrices with non-zero subdiagonal elements are simple), with eigenvectors given by

$$
u_{1}=\left(\begin{array}{l}
1 \\
1 \\
1
\end{array}\right), \quad u_{2}=\left(\begin{array}{c}
-1 \\
0 \\
1
\end{array}\right), \quad u_{3}=\left(\begin{array}{c}
1 \\
-2 \\
1
\end{array}\right),
$$

represented in Figure 1. Thus we see that in the only nodal domain of $\tilde{u}_{1}$ there is exactly one zero of $\tilde{u}_{2}$, and in each nodal domain of $\tilde{u}_{2}$ there is exactly one zero of $\tilde{u}_{3}$ (both of them being on an edge).

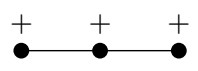

$u_{1}$

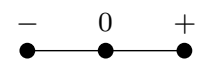

$u_{2}$

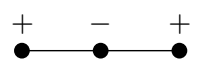

$u_{3}$

FIGURE 1. Eigenvectors of a linear graph with weights 1,1 . 


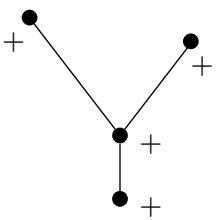

$u_{1}$

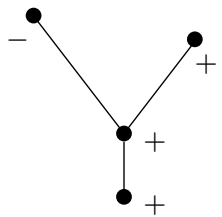

$u_{2}$

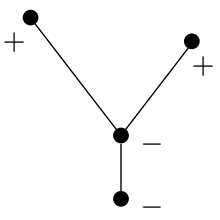

$u_{3}$

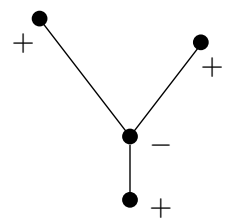

$u_{4}$

Figure 2. Eigenvectors of a star graph with weights $1, \frac{1}{4}, \frac{1}{3}$.

Consider now the tree which is a star graph with four vertices and weights $1, \frac{1}{4}, \frac{1}{3}$. The associated Laplacian is given by

$$
\mathcal{L}=\left(\begin{array}{cccc}
1 & -1 & 0 & 0 \\
-1 & \frac{19}{12} & -\frac{1}{4} & -\frac{1}{3} \\
0 & -\frac{1}{4} & \frac{1}{4} & 0 \\
0 & -\frac{1}{3} & 0 & \frac{1}{3}
\end{array}\right)
$$

and the eigenvalues are $0, \frac{4}{3}-\frac{\sqrt{10}}{3}, \frac{1}{2}, \frac{4}{3}+\frac{\sqrt{10}}{3}$ and are simple. The eigenvectors are represented in Figure 2 and can be seen to have no zero vertices. Again one can see the interlacing property, that is in each nodal domain of $\tilde{u}_{i}$ there is exactly one zero of $\tilde{u}_{i+1}$.

To show that assumption (A) is essential, we provide the following counterexample, represented in Figure 3. Consider the star graph with four vertices, and weights $\frac{1}{2}, 1,1$. The ordered eigenvalues of the associated Laplacian are $0,2-\sqrt{2}, 1,2+\sqrt{2}$, and in particular they are simple. The corresponding eigenvector for the third eigenvalue 1 is $u_{3}={ }^{t}(0,0,-1,1)$ and has a zero graph, hence its linear extension is identically zero on one of the nodal domains of $\tilde{u}_{2}$, which fails Theorem 4.1. Note also that $u_{3}$ has only $2(<3)$ strong sign graphs.

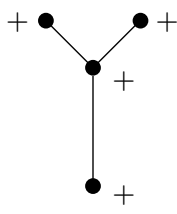

$u_{1}$

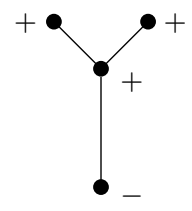

$u_{2}$

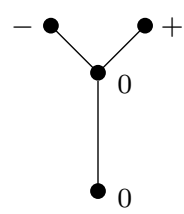

$u_{3}$

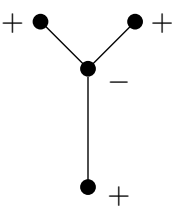

$u_{4}$

FiguRE 3 . The star graph with weights $\frac{1}{2}, 1,1$ counterexample: $u_{3}$ has a zero graph and only two strong sign graphs.

Counterexamples of eigenvectors associated with non-simple eigenvalue $\lambda_{n}$ having more than $n$ strong sign graphs can be found in [7. For instance, one can show that the second eigenvalue of the Laplacian associated with a star graph with $N$ vertices with weight 1 on each edge has multiplicity $N-2$, with an eigenvector having only a zero vertex at the unique internal vertex (of degree $N-1$ ), and hence having $N-1$ strong sign graphs, see [7] for more details. 


\section{GREen's Formula}

Analogues of Green's formula on graphs have been already considered, see for instance [4. To be self-contained we give a straightforward proof in our context.

Let us first introduce some boundary sets. Let $u$ be some function on $V$. Let $G$ be a strong positive sign graph of $u$, and $\tilde{G}$ the corresponding nodal domain of $\tilde{u}$. Define the following boundary sets:

$$
\begin{aligned}
\delta(G) & =\{x \in V \backslash G \mid x \sim y, \text { for some } y \in G\}, \\
\partial(G) & =\{(x, y) \in E \mid x \in G, y \in \delta(G)\} .
\end{aligned}
$$

Since $u(x)>0$ for all $x \in G$, we have $u(y) \leq 0$ for all $y \in \delta(G)$, otherwise $y$ would be in $G$. This implies that $\tilde{u}_{x y}$ vanishes on $\left.] 0, l(x, y)\right]$, where $(x, y)$ is an edge belonging to $\partial(G)$. Let us call $t(x, y)$ the point of $] 0, l(x, y)]$ where $\tilde{u}_{x y}$ vanishes. We have

$$
t(x, y)=\frac{l(x, y) u(x)}{u(x)-u(y)} .
$$

The boundary of $\tilde{G}$ (depending on $u$ ) is then defined by

$$
\mathcal{B}(\tilde{G})=\{t(x, y) \mid(x, y) \in \partial(G)\} .
$$

This boundary set is defined in the same way for $G$ a strong negative sign graph. Let $\nabla$ be the usual gradient on $\mathbb{R}$. We have the analogue of the Green's formula:

Proposition 3.1 (Green's formula). Let $\mathcal{A}=\mathcal{L}+r$ be a Schrödinger operator. Let $u, v$ be functions on $V$, and $\tilde{u}, \tilde{v}$ their linear extensions on edges. Let $G$ be a strong sign graph of $u$, and $\mathcal{B}(\tilde{G})$ the boundary of the corresponding nodal domain of $\tilde{u}$. Then, we have,

$$
\sum_{x \in G} \mathcal{A} u(x) v(x)-\sum_{x \in G} u(x) \mathcal{A} v(x)=-\sum_{t \in \mathcal{B}(\tilde{G})} \nabla \tilde{u}(t) \tilde{v}(t) .
$$

Proof. Since,

$$
\sum_{x \in G} \mathcal{A} u(x) v(x)-\sum_{x \in G} u(x) \mathcal{A} v(x)=\sum_{x \in G} \mathcal{L} u(x) v(x)-\sum_{x \in G} u(x) \mathcal{L} v(x),
$$

it suffices to prove the proposition for the Laplacian $\mathcal{L}$.

We have,

$$
\begin{aligned}
\sum_{x \in G} \mathcal{L} u(x) v(x)=\sum_{x \in G} \sum_{\substack{y \in G \\
y \sim x}} c(x, y)(u(x)-u(y)) v(x) & \\
& +\sum_{\substack { x \in G \\
\begin{subarray}{c}{y \in \delta(G) \\
y \sim x{ x \in G \\
\begin{subarray} { c } { y \in \delta ( G ) \\
y \sim x } }\end{subarray}} c(x, y)(u(x)-u(y)) v(x) .
\end{aligned}
$$

But,

$$
\begin{aligned}
\sum_{x \in G} \sum_{\substack{y \in G \\
y \sim x}} c(x, y)(u(x)-u(y)) v(x) & \\
= & \frac{1}{2} \sum_{x \in G} \sum_{\substack{y \in G \\
y \sim x}} c(x, y)(u(x)-u(y))(v(x)-v(y)),
\end{aligned}
$$


and the same holds by exchanging the roles of $u$ and $v$. Thus, we obtain

$$
\begin{aligned}
\sum_{x \in G} \mathcal{L} u(x) v(x)-\sum_{x \in G} \mathcal{L} v(x) u(x)=\sum_{x \in G} \sum_{\substack{x \in \delta(G) \\
y \sim x}} c(x, y)(u(x)-u(y)) v(x) & -\sum_{x \in G} \sum_{\substack{y \in \delta(G) \\
y \sim x}} c(x, y)(v(x)-v(y)) u(x) .
\end{aligned}
$$

On the interval $[0, l(x, y)]$, we have $\tilde{v}(t)=\frac{v(y)-v(x)}{l(x, y)} t+v(x)$, hence

$$
\tilde{v}(t(x, y))=-\frac{v(x)-v(y)}{u(x)-u(y)} u(x)+v(x),
$$

for $t(x, y)=\frac{l(x, y) u(x)}{u(x)-u(y)} \in \mathcal{B}(\tilde{G})$. Furthermore, since $\nabla$ is the usual derivate, we have

$$
\nabla \tilde{u}(t)=\frac{u(y)-u(x)}{l(x, y)}=-c(x, y)(u(x)-u(y)),
$$

for all $t \in[0, l(x, y)]$. Hence, we have

$$
\begin{aligned}
\sum_{t \in \mathcal{B}(\tilde{G})} \nabla \tilde{u}(t) \tilde{v}(t)= & \sum_{x \in G} \sum_{\substack{y \in \delta(G) \\
y \sim x}} \nabla \tilde{u}(t(x, y)) \tilde{v}(t(x, y)) \\
= & \sum_{x \in G} \sum_{\substack{y \in \delta(G) \\
y \sim x}} c(x, y)(v(x)-v(y)) u(x) \\
& \quad-\sum_{x \in G} \sum_{\substack{y \in \delta(G) \\
y \sim x}} c(x, y)(u(x)-u(y)) v(x),
\end{aligned}
$$

so the proposition is proved.

\section{INTERLACING PROPERTY OF ZEROS OF EIGENVECTORS}

As in the classical result of Courant, we can now prove the interlacing property of the zeros of eigenvectors of $\mathcal{A}$.

Theorem 4.1. Let $\Gamma$ be a finite tree with $N$ vertices, and $\mathcal{A}$ a Schrödinger operator on $\Gamma$. Let $\lambda_{n}, n=1, \ldots, N$, be the ordered eigenvalues of $\mathcal{A}$, and let $u_{n}$ be an eigenvector associated with $\lambda_{n}$, and denote by $\tilde{u}_{n}$ its linear extension. If the zero vertices of the eigenvectors $u_{n}$ 's are of degree at most two, then the zeros of the $\tilde{u}_{n}$ 's interlace, in the sense that in any nodal domain of $\tilde{u}_{n-1}$ there is exactly one zero of $\tilde{u}_{n}$.

Proof. Recall that we have seen that by Fiedler $[\underline{8}$ if the zero vertices of the eigenvectors of $\mathcal{A}$ are of degree at most two, then the eigenvalues of $\mathcal{A}$ are simple and the corresponding eigenvectors are without a zero graph.

Let $\lambda$ and $\mu$ be two eigenvalues of $\mathcal{A}$ with $\lambda<\mu$, and let $u$ and $v$ be the associated eigenvectors. Let $G$ be a strong positive sign graph of $u$, and $\tilde{G}$ be the corresponding nodal domain of $\tilde{u}$, with boundary $\mathcal{B}(\tilde{G})$. Suppose that $\tilde{v}$ does not change sign on $\tilde{G}$, and say $\tilde{v}>0$ on $\tilde{G}$. This implies that $v(x)>0$, for all $x \in G$. By Green's formula, we have

$$
(\lambda-\mu) \sum_{x \in G} u(x) v(x)=-\sum_{t \in \mathcal{B}(\tilde{G})} \nabla \tilde{u}(t) \tilde{v}(t) .
$$


The left hand side of the above expression is then negative. Since $\nabla \tilde{u}=c(x, y)(u(y)-$ $u(x))<0$ on the edges $(x, y) \in \partial G$, and $\tilde{v}>0$ on $\tilde{G}$, then the right hand side is non-negative. So there is a contradiction, and $\tilde{v}$ must vanish and thus change sign on $\tilde{G}$ since a zero of $\tilde{v}$ is between two opposite sign vertices, even if the zero is a vertex since we cannot have zero graphs by assumption. Using the same argument on all of the sign graphs of $u$, we conclude that $\tilde{v}$ has at least one more nodal domain than $\tilde{u}$. By the discrete nodal theorem of [7, since all eigenvalues $\lambda_{i}$ of $\mathcal{A}$ are simple, then $u_{n}$ has at most $n$ strong sign graphs, and hence $\tilde{u}_{n}$ has at most $n$ nodal domains. So, we deduce from this by iteration, that $\tilde{u}_{n}$ has exactly $n$ nodal domains, and hence $u_{n}$ has exactly $n$ strong sign graphs.

Since on a tree, there is a unique path connecting any two points of the tree, the different nodal domains of $\tilde{u}_{n}$ are separated by a unique zero of $\tilde{u}_{n}$. Since $\tilde{u}_{n}$ has exactly $n$ nodal domains separated by its zeros, $\tilde{u}_{n}$ has exactly $n-1$ zeros in $\Gamma$. Hence, since $\tilde{u}_{n}$ must vanish in the interior of every nodal domain of $\tilde{u}_{n-1}$, the zeros of the $\left(\tilde{u}_{n}\right)_{1 \leq n \leq N}$ must interlace in the sense that in any nodal domain of $\tilde{u}_{n-1}$, there is exactly one zero of $\tilde{u}_{n}$.

Acknowledgments. I would like to thank Gregory Berkolaiko for some useful comments and suggestions.

\section{REFERENCES}

[1] Ram Band, Idan Oren, and Uzy Smilansky, Nodal domains on graphs-how to count them and why?, Analysis on graphs and its applications, Proc. Sympos. Pure Math., vol. 77, Amer. Math. Soc., Providence, RI, 2008, pp. 5-27.

[2] Gregory Berkolaiko, A lower bound for nodal count on discrete and metric graphs, Comm. Math. Phys. 278 (2008), no. 3, 803-819.

[3] Türker Biylkoğlu, A discrete nodal domain theorem for trees, Linear Algebra Appl. 360 (2003), 197-205.

[4] Türker Bıyıkoğlu, Josef Leydold, and Peter F. Stadler, Laplacian eigenvectors of graphs, Lecture Notes in Mathematics, vol. 1915, Springer, Berlin, 2007, Perron-Frobenius and FaberKrahn type theorems.

[5] Galya Blum, Sven Gnutzmann, and Uzy Smilansky, Nodal Domains Statistics: A Criterion for Quantum Chaos, Phys. Rev. Lett. 88 (2002), no. 11, 4.

[6] R. Courant and D. Hilbert, Methods of mathematical physics. Vol. I, Interscience Publishers, Inc., New York, N.Y., 1953.

[7] E. Brian Davies, Graham M. L. Gladwell, Josef Leydold, and Peter F. Stadler, Discrete nodal domain theorems, Linear Algebra Appl. 336 (2001), 51-60.

[8] Miroslav Fiedler, Eigenvectors of acyclic matrices, Czechoslovak Math. J. 25(100) (1975), no. 4, 607-618.

[9] Joel Friedman, Some geometric aspects of graphs and their eigenfunctions, Duke Math. J. 69 (1993), no. 3, 487-525.

[10] Sven Gnutzmann, Panos D. Karageorge, and Uzy Smilansky, Can one count the shape of a drum?, Phys. Rev. Lett. 97 (2006), no. 9, 4.

[11] Alexander R. Griffing, Benjamin R. Lynch, and Eric A. Stone, An eigenvector interlacing property of graphs that arise from trees by Schur complementation of the Laplacian., Linear Algebra Appl. 438 (2013), no. 3, 1078-1094.

[12] Idan Oren and Ram Band, Isospectral graphs with identical nodal counts, J. Phys. A 45 (2012), no. 13, 135203, 12.

[13] Hao Xu and Shing-Tung Yau, Nodal domain and eigenvalue multiplicity of graphs, J. Comb. 3 (2012), no. 4, 609-622.

Université Paul Sabatier, Institut de Mathématiques de Toulouse, 118 route de NarBonne, F-31062 Toulouse Cedex 9

E-mail address: francois.chapon@math.univ-toulouse.fr 